\title{
Contents, Vol. 92, 1937
}

Inhaltsverzeichnis.

Eigenarbeiten. Seit $\beta$

Custodis, Seltene Augenkomplikationen bei Infektionskrank-

heiten (Diphtherie, Scharlach, Masern) 336

Davids, Herm., Über Augenerkrankungen bei Agranulocytose 193 Fńede, B., Über Absaugung weicher Stare und Starreste. Verwendung des Starsaugers bei Hyphaema, Hypopyon

und Keratoplastik 201

Gardilcic, Ante, Perineuritis und Periarteriitis ciliaris bei

einem frischen Fall von Herpes zoster op $\cap$ thalmicus . 35 Hamburger, F. A., Überzählige

Bildungen von abnorm ge-

lagerten Tränenröhrchen

Hausmann, Gertrud, Zur Kenntnis der Dauerresultate der

Zyklodialyse 139

Johansson, Ernst, Zur Ätiologie der Iridocyclitis .... 154 Karsch, ]', Zur Klinik und Erbbiologie des angeborenen

Stars, insbesondere des Schichtstars 322

Klar, B., Ein Beitrag zur Pathogenese des Herpes corneae (unter Zugrundelegung des

Krankengutes der Bonner

Augenklinik) 215

Paas, W. L., Doppelseitiges Glaukom bei Leontiasis ossea 221 Procksch, Marie, Über

Operationsaussichten bei komplizier-

ter Cataract 129

Beis, W., Die Darstellung des Auges in der expressionisti-

schen Kunst 158

Beiser, K. A., Über grippöse Hämorrhagien am Augenhinter-

grund 207

Bintelen, F., Über elephantiastische Lidveränderungen, zu-gleich ein Beitrag zur Kenntnis des osteo-dermopathi-

schen Syndroms: Touraine-Solente-Golé 1

-, Zur Histologie des submakularen senilen Pseudotumors (Scheibenförmige Entartung der

Netzhautmitte: Junius

und Kuhnt) 306

Bollin, J. L., Katarakt bei Neurodermie

Salzmann, Maximilian, Die intrasklerale Epithelzyste . . . 275

Schmidt, Nachruf auf Prof. Römer 273

$\S$ panic, A., Ein seltener Fall von nichtexulzerierter Binde-hautsklerose der Übergangsfalte des Unterlides, derhalbmondförmigen Falte und des Augapfels. (Beitragzur experimentellen parenchymatösen Keratitis). (HierzuTafel I) 300

IV Inhaltsverzeichnis. 


\section{Seit $\theta$}

Pick, Zur Behandlung degenerativer Hornhauterkrankungen 230

Berichte iiber die ophthalmologische Literatur.

Bakteriologie, Parasitologie, Immunitätsforschung und Ver-giftungen. (Berichtsjahr Juli 1935 bis Juni 1936.) VonProf. Dr. A. Pillat-G $\tau a, z \quad 42$

Verletzungen - Versicherungswesen und soziale Medizin -Blindenwesen. (Berichtsjahr vom I. Januar bis 31. De-zember 1936.) Von Dr. B. Heßberg-Essen 81

Vegetative Physiologie und Pathologie. (Berichtsjahr vomI. Oktober 1935 bis 30. September 1936.) Von DozentDr. Walter Bauh-Leipzig 96

Entstehung und Klinik der Refraktionsanomalien, Seh-schärfe, Akkommodation. (Berichtszeit

vom I. Juli 1934bis 30. September 1936.) Von Dr. Frieärich Bamach-Wien168

Kornea, Sklera, Episklera. (Berichtsjahr vom 1. März 1936

bis I. März 1937.) Von Prof. Dr. Hans Schmelzer-E $\tau$ lã.ngen 232

Iris und Ziliarkörper. Von Prof. Dr. K. Velhagen-iíalle . 346

Glaukom. Von F. Bintelen-Basel 350

Gesellschaftsberichte.

Elite Tagung der Bayerischen Augenärztlichen Vereinigung in Würz-

burg am 6. Dezember 1936174

Erste Tagung märkischer Augenärzte in der Universitäts-Augen-

klinik Berlin, 5. und 6. Dezember $1936 \quad 250$

Ophthalmologische Gesellschaft in Wien:

Sitzung vom 18. Januar 1937112 Sitzung vom 15. Februar 1937247 Verhandlungen der

Schwedischen Gesellschaft der Augenärzte:

Sitzung vom 23. November 1935117

Sitzung vom 29. Februar 1936. 119

Jahresversammlung in Stockholm am 24. Mai 1936120

Diagnose und Therapie $124,187,267,369$

Buchbesprechungen 127, 191, 372

XV. Internationaler Ophthalmologen-Kongreß, Eairo

Berichtignng zu dem Bericht: Lider, Tränenorgane, Bindehaut

Bd. 90, S. 344191

Personalien 128

S. Sachregister zu Band $92 \quad 374$

Namenregister zu Band $92 \quad 379$ 